\title{
Engineering Behavior of Concrete with Recycled Aggregate
}

\author{
Afizah Ayob ${ }^{1, a}$, Mohd. Ekhwan Razali ${ }^{1}$, Salina Alias ${ }^{2}$, Abdul Ghapar Ahmad ${ }^{1}$, Dayang Siti Hazimmah Ali $^{3}$ \\ ${ }^{1}$ Department of Civil Engineering, School of Environmental Engineering, Universiti Malaysia Perlis, 02600 Arau, Perlis, Malaysia \\ ${ }^{2}$ Faculty of Civil Engineering, Kampus Permatang Pauh, UiTM Pulau Pinang, 03500 Permatang Pauh, Pulau Pinang, Malaysia \\ ${ }^{3}$ School of Engineering and Technology, University College of Technology Sarawak, 868 Persiaran Brooke, 96000 Sibu, Sarawak, \\ Malaysia
}

\begin{abstract}
Concrete is extensively used as construction materials in Malaysia. Concrete contributes suitable feature for construction industry for instance durability, adequate compressive strength, fire resistance, availability and is economic as compared to other construction materials. Depletion of natural resources and disposal of construction and demolition waste remarkably claim environmental threat. In this paper, the engineering behavior, durability, and concrete microstructure of recycled concrete aggregates (RCA) on short-term concrete properties were investigated. The studied concrete at design mix proportion of 1:0.55:2.14:2.61 (weight of cement :coarse aggregates :sand :water) used to obtain medium-high compressive strength with $20 \%, 50 \%$, and $100 \%$ of RCA. Results show that for the same water/cement ratio, RCA replacement up to $50 \%$ still achieved the targeted compressive strength of $25 \mathrm{MPa}$ at 28 curing days. Addition, at similar RCA replacement, the highest carbonation depth value was found at $1.03 \mathrm{~mm}$ which could be attributed to the pozzolanic reaction, thus led to lower carbonation resistance. Scanning electron microscopy microstructure shows that the RCA surface was porous and covered with loose particles. Moreover, the interfacial transition zone was composed of numerous small pores, micro cracks, and fissures that surround the mortar matrix. On the basis of the obtained results, recommendable mineral admixtures of RCA are necessary to enhance the quality of concrete construction.
\end{abstract}

\section{Introduction}

A large amount of construction and demolition waste (CDW) is being generated by the construction industry in Malaysia due to demands of small and major infrastructure projects, that includes commercial building, and housing development planning. Nonusable and inert waste is the result of enormous construction and demolition activities. It consists abundantly of concrete, bricks and block, aggregate, soil and sand, stones, pebbles, graded organic particles and wood, among others. Providing land fill sites for CDW is not the main solution for this problem. Technology and science must play a main role in fostering the successful implementation of waste management. Factors, such as environmental sustainability, economic condition, land fill space depletion, and quality of construction basic raw material, are essential for recycling/reusing CDW [1]. Most CDW in Malaysia was formerly assumed nonusable and illegally discarded. To date, these wastes are totally utilize as recycled/reused concrete aggregates and are benefited for various applications in construction $[2,3]$.

Recycled concrete aggregate (RCA) is an environmentally friendly material that employ CDW, especially demolished concrete waste as alternative aggregates to take place of natural aggregates. This RCA is produced partly or totally by processes of cleaning, pulverizing, classifying and proportional mixing. However, the application is complex because the properties of RCA are different from natural aggregates. In addition, the quality of RCA varies among different sources. The physical properties of RCA mainly determine on water absorption, adhered mortar quality, amount of adhered mortar, contaminant, chemical constituent and the interfacial transition zone (ITZ) between the natural aggregates and original cement mortar. Adhered mortar is a porous material, and its porosity rely on the originally selected ratio of water to cement $(w / c)$ [3, 4]. The high porosity of RCA is responsible for its low resistance toward mechanical and chemical actions. The quantity of adhered mortar significantly increases with decreasing aggregate size. RCA exhibits lower bulk density and greater water absorption than natural aggregates because of the adhered mortar amount. Meanwhile, ITZ is the weakest section in concrete due to its less porosity and crack resistance than either hardened cement paste or aggregate [3]. Crushing procedure usually affects the amount of adhered mortar and ITZ in the concrete behavior with recycled aggregate.

RCA application remains unfavorable, particularly in countries where natural aggregates are still abundant. RCA replacement in concrete is inversely associated to its workability. Several works have been conducted to investigate the essential properties of RCA, such as compressive strength, flexural strength, tensile strength, elastic modulus, and durability. The actual concrete strength differs from compressive strength of the original concrete used as recycled aggregates. The reduction in compressive strength is not prominent, when the RCA replacement is relatively up to $30 \%$ [5]. The compressive strength of a recycled aggregate concrete becomes unsatisfactory when high RCA percentage is used to substitute natural aggregates. However, a suitable concrete mix design enables the RCA to achieve target 
strength in wide range of application in construction. Kwan et al. (2012) [2] achieved target strength even when $80 \%$ of the total coarse aggregate content was replaced by RCA. The same authors have reported that, even though the porous materials of adhered mortar could significantly increase the permeability, the curing period would control the intrinsic permeability of RCA for the long term durability of the concrete structure.

The concept of reusing and utilizing RCA from CDW has been used since the World War II in Europe as unbound sub-base or main load-bearing layer material of the pavement [5]. Natural aggregates cannot be reproduced in a short period of time, and aggregates basically hold $60 \%$ to $75 \%$ of the concrete volume; thus no doubt RCA is suggested for construction applications. The use of RCA not only solves the absence of natural resources but also saves land disposal for CDW. Therefore, this study aims to report the engineering behavior of RCA in a concrete construction mixture. Engineering properties, durability, and concrete microstructure of hardened concrete used as replacement of natural aggregates with RCA were discussed.

\section{Materials and Methods}

\subsection{Materials}

Ordinary Portland cement (OPC) with a specific gravity of 3.24 was employed as the main binder for the experiment. Table 1 shows the typical chemical constituent of OPC.

Natural coarse aggregates were crushed granite with sizes of 4.75 to $20 \mathrm{~mm}$ were obtained from local quarries. The aggregates were washed with water to remove any unwanted particles, such as clay, dirt, and dust, and were then air dried. Fine aggregates (with less than $5.0 \mathrm{~mm}$ size) used were uncrushed quartzite natural river sand. RCA was obtained from a demolished building (Fig. 1) and was manually crushed to achieve the required grain size distribution of $10-20 \mathrm{~mm}$.

Table 1. Major chemical constituents of OPC

\begin{tabular}{|c|c|}
\hline Constituent of OPC & \% \\
\hline Lime $(\mathrm{CaO})$ & 68.85 \\
\hline Silica $\left(\mathrm{SiO}_{2}\right)$ & 15.00 \\
\hline Alumina $\left(\mathrm{Al}_{2} \mathrm{O}_{3}\right)$ & 4.30 \\
\hline Sulfur trioxide $\left(\mathrm{SO}_{3}\right)$ & 4.00 \\
\hline Iron oxide $\left(\mathrm{Fe}_{2} \mathrm{O}_{3}\right)$ & 3.96 \\
\hline Potassium oxide $\left(\mathrm{K}_{2} \mathrm{O}\right)$ & 1.08 \\
\hline Magnesia $(\mathrm{MgO})$ & 1.30 \\
\hline Barium oxide $(\mathrm{BaO})$ & 0.34 \\
\hline Titanium dioxide $\left(\mathrm{TiO}_{2}\right)$ & 0.34 \\
\hline Others & 0.73 \\
\hline
\end{tabular}

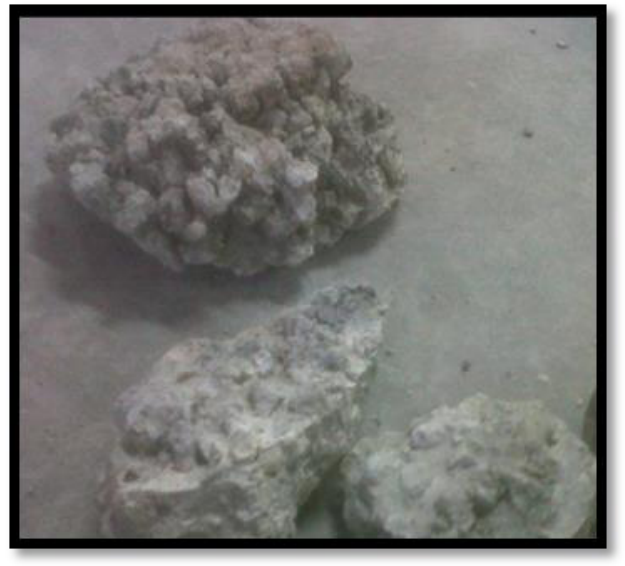

Figure 1. (a) RCA samples from CDW

Grain sizes of aggregates were reported to influence density and water adsorption. Aggregate size distribution was determined in accordance with BS 882 (1992) [6], and the result of sieve analysis shown in Table 2 . Given the high water absorption capacity of RCA, aggregates were maintained in a saturated surface dry condition before the start of the mixing process.

Table 2. Aggregate size distribution

\begin{tabular}{|l|l|}
\hline Type of Aggregates & Grain size (mm) \\
\hline Fine & $0-5$ \\
\hline Natural coarse & 4.75 to 20 \\
\hline \multirow{2}{*}{ RCA } & $4-8$ \\
\cline { 2 - 2 } & $8-20$ \\
\hline
\end{tabular}

\subsection{Concrete Mixes}

Four types of mixtures were prepared by replacing the coarse aggregate with $20 \%, 50 \%$ and $100 \%$ of RCA. The percentage of replacement was determined based on the total weight of the coarse aggregate content. Normal specimen was denoted as $0 \%$ of RCA replacement. The replacement of natural coarse aggregate with RCA is expected to increase remarkably the $\mathrm{w} / \mathrm{c}$ ratio [7]. Thus, the design mix proportion was set up as shown in Table 3 [8] for design targeted compressive strength of $25 \mathrm{MPa}$ at 28 curing days. All specimens were casted in $150 \mathrm{~mm}$ $\times 150 \mathrm{~mm} \times 150 \mathrm{~mm}$ molds under laboratory condition for $28 \mathrm{~h}$, demolded, and then completely submerged in water for curing until testing.

Table 3. The design mix proportion

\begin{tabular}{|l|c|}
\hline \multicolumn{1}{|c|}{ Constituents } & $\begin{array}{c}\text { Proportion } \\
\left(\mathbf{k g} / \mathbf{m}^{\mathbf{3}} \mathbf{)}\right.\end{array}$ \\
\hline Cement & 418 \\
\hline Natural coarse aggregates /RCA & 987 \\
\hline Fine aggregate & 745 \\
\hline Water & 2.3 \\
\hline
\end{tabular}

The water absorption and compressive strength tests were performed according to BS EN 12390-3 (2009) [9] and BS 1881-122 (2011) [10], respectively, by using three cubes to obtain the average value of the specimens. 
Carbonation test was conducted to investigate the concrete durability [11]. Carbonation depth was measured after spraying a solution of phenolphthalein on a freshly broken concrete surface (Fig. 2 (a)). The carbonated region with decreased alkalinity remained colorless, whereas the uncarbonated region with high alkalinity turned its color to pink. Examples of six spots reading location are shown in Fig. 2 (b).

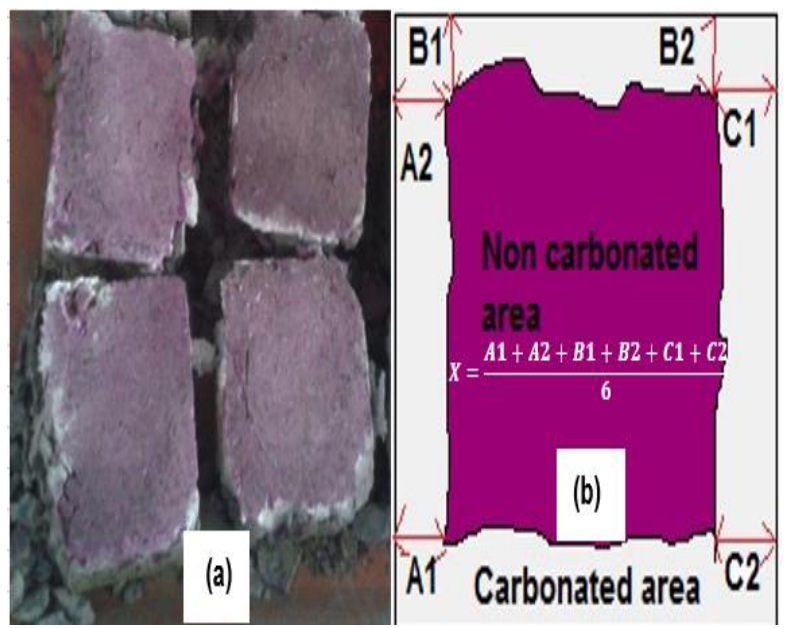

Figure 2. (a) Phenolphthalein sprayed on dried freshly broken concrete surface; (b) readings location spot of carbonation depth $(X)$

The concrete microstructure properties on the RCA surface were observed after 28 curing days under a scanning electron microscope (SEM; JEOL JSM 6460 LA).

\section{Results and discussion}

\subsection{Water absorption}

Fig. 3 shows the influence of RCA content on water absorption rate. The water absorption rates of concrete containing the RCA were higher than that of the normal concrete. In this study, the water absorption rate of natural course aggregate recorded at $2.6 \%$. Water absorption rate of concrete shows and increasing profile in relation to higher RCA content (Fig. 3).

The highest water absorption value was obtained by $100 \%$ RCA specimen, which is 0.6 times higher than that of normal. It was due to the higher absorption capacity of the RCA itself resulting from cement mortar remains attaching to its surface, and the substitution level of RCA, which then has developed higher osmosis pressure within the concrete matrix, that imposes a high risk towards the durability of concrete.

At the same time, the RCA is a porous material that correlates to high permeability properties for the concrete and greater probability of interconnection between the micro structure systems of the specimen. Additionally, the pressure-induced flow would contribute the easiest way for the transmission of molecules as the internal flow paths are not blocked.

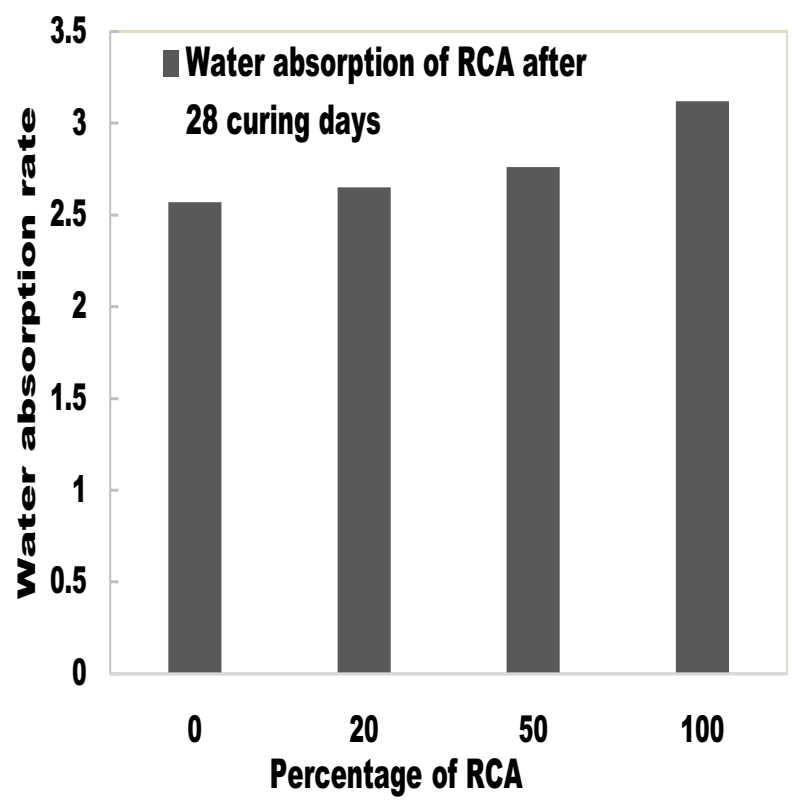

Figure 3. Behavior of the water absorption rate related to RCA content after 28 curing days

These reveal the highest water absorption at $100 \%$ RCA sample that occurs through the capillary effects by the high osmosis pressure when the dry samples were submerged into the water.

\subsection{Compressive strength}

Fig. 4 shows the influence of RCA content on the average results of concrete compressive strength after 7 and 28 curing days. The figure shows the similar profile of strength development as the normal concrete, where the compressive strength increased with the number of curing days for $20 \%$ and $50 \%$ of RCA replacement. This result was due to the increased amount of hydration products produced during prolonged curing period. The RCA content in concrete greatly affected the compressive strength of the specimen. The normal concrete $(0 \%$ RCA) achieved the highest strength, followed by $20 \%, 50 \%$, and $100 \%$ of RCA replacement (Fig. 4).

The results indicate a decreasing trend in compressive strength toward high RCA content for 7 and 28 curing days. This finding was consistent to that of Kwan et al. (2012) [2] who reported that RCA influences concrete design and durability. This trend was not observed by Lotfy and Al-Fayez, (2015) [13] when they used high-quality RCA produced on a commercial scale. Their RCA performed equally to virgin aggregates up to $30 \%$ coarse replacement and $20 \%$ granular replacement. 


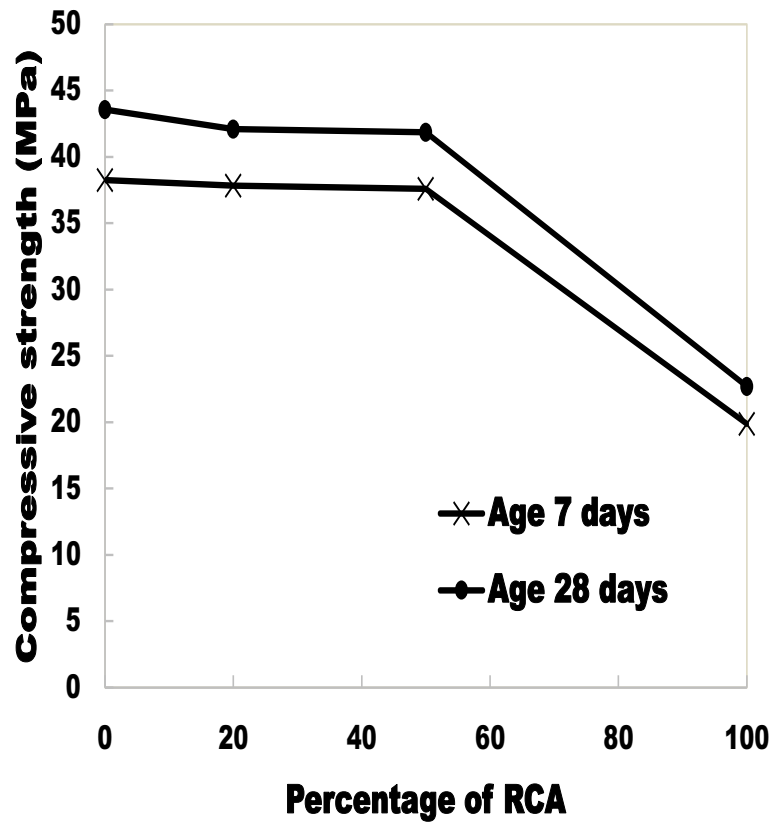

Figure 4. Behavior of the concrete compressive strength related to RCA content for age 7 days and 28 days

The inverse trend between the RCA content and compressive strength was probably due to the poor quality of the adhered mortar, which underwent crushing process, that weakened the ITZ in the concrete. The presence of adhered residual cement mortar on the RCA surface was in accordance to porous, rough [2] and weak interfacial bond strengths between the aggregate and the presence of micro cracks and fissures, which could decrease the bonding with the surrounding matrix [5]. Therefore, by giving ultimate load to the concrete, the weak sections would have greater probability to be interconnected between each other as the RCA content increases. The new ITZ between the RA and cement paste waste was highly efficient. This ITZ exhibited a lower aggregate $\mathrm{w} / \mathrm{c}$ ratio than the adhered mortar on the RCA surface. This ITZ is thus denser than the old cement paste, which was accordingly weaker [14].

In addition, the RCA has high hydrated and nonhydrated levels that can increase its saturation level and then later affect the concrete strength. At higher saturation levels, the mechanical bonding between the cement paste and RCA became weaker, which led to low compressive strength, as observed in $100 \%$ of RCA replacement (Fig. 4). Thereby, replacing the coarse aggregate with up to $50 \% \mathrm{RCA}$ is the optimum level for the mix proportion because of the absence of significant reduction in the compressive strength.

\subsection{Carbonation depth}

The results of carbonation depths in specimens with varied RCA contents throughout exposure time are shown in Fig. 5. A high influence of RCA content was observed in all studied admixtures. However, for different curing days, the incorporation of RCA had no significant effect on carbonation depth.

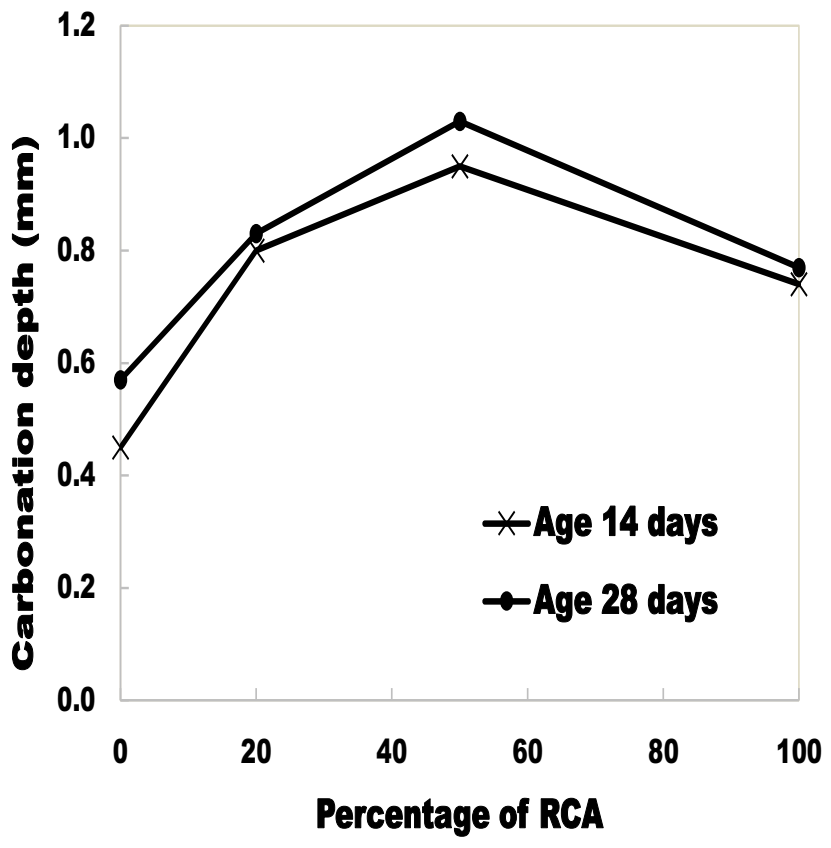

Figure 5. Behavior of carbonation depth related to the RCA content for age 14 days and 28 days

The highest difference of carbonation depth value was found in $50 \%$ RCA replacement, which depicted almost similar behavior at 14 and 28 curing days. The measured carbonation depths for normal samples were 0.45 and $0.57 \mathrm{~mm}$, and the depths increased 0.95 and $1.03 \mathrm{~mm}$ with $50 \%$ RCA replacement, respectively, at 14 and 28 curing days. The negative affect of $50 \%$ RCA in carbonation depth could be attributed to the pozzolanic reaction. The pozzolanic material in the concrete specimen consumes calcium hydroxide $(\mathrm{CH})$ and hydrated calcium silicate (CS). Calcium hydroxide and hydrated calcium silicate are the main hydration products of the adhered mortar on RCA. $\mathrm{CO}_{2}$ can penetrate into the pores of the adhered mortar and react with the reaction products as follows:

$$
\begin{aligned}
& \mathrm{Ca}(\mathrm{OH})_{2}+\mathrm{CO}_{2} \longrightarrow \mathrm{CaCO}_{3}+\mathrm{H}_{2} \mathrm{O} \\
& \mathrm{C}-\mathrm{S}-\mathrm{H}+\mathrm{CO}_{2} \longrightarrow \mathrm{CaCO}_{3}+\mathrm{SiO}_{2} \cdot \mathrm{nH}_{2} \mathrm{O}
\end{aligned}
$$

Carbonation of $\mathrm{CH}$ and $\mathrm{C}-\mathrm{H}-\mathrm{S}$ (calcium silicate hydrates) provides $11.0 \%$ and $23.1 \%$ of solid volume, respectively [15], therefore, carbonation could decrease the porosity of the adhered mortar and consequently reduce the alkalinity of concrete $[16,17]$. At the same time, C-H-S formed during hydration could contribute a bridging effect within the structure that wraps the aggregate. Subsitutely, the dissolved silicates could seep through the wrap and precipitate as an outer layer, thus generating a dense micro structures and decreasing the number of pores. In addition, carbonation enhancement of RCA, which is based on the chemical reaction between $\mathrm{CO}_{2}$ cement minerals and hydration product, promoted the adhesion of mortars, as well the original and new ITZs around the RCA surface. 
Carbonation depth was affected by water adsorption, crushing value of RCA [4] alkalinity of binding medium, and aggregate size. In this case, the aggregate size for $50 \%$ RCA replacement showed higher probability that the concrete mixes that composed of fine aggregates exhibited higher carbonation depths than those of large RCA. The greater porosity of fine RCA was due to the greater amount of adhered mortar. The carbonation development of $100 \%$ RCA replacement decreased to 0.74 and $0.77 \mathrm{~mm}$ over time for 14 and 28 curing days, respectively (Fig. 5).

\subsection{RCA microstructure}

Fig. 6 shows the microstructure of RCA samples after 28 curing days. The angular conventional concrete shape has cleaner surface aggregate, and is less porous and free from loose particles, such as dust (Fig. 6(a)). The microstructures of the concrete with $20 \%$ RCA content and the conventional concrete were not significantly different (Fig. 6(b) (c)).

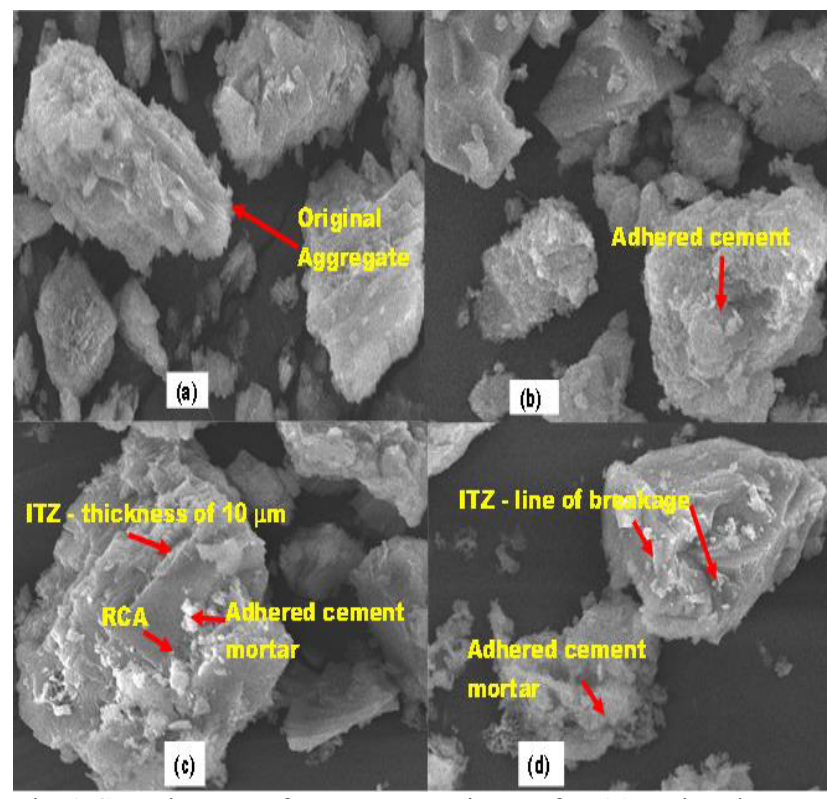

Fig.6. SEM images of concrete specimen after 28 curing days (a) $0 \%$ RCA content; (b) $20 \%$ RCA content; (c) $50 \%$ RCA content; (d) 100\% RCA content.

Meanwhile, the angular shape and rough surface of concrete with $50 \%$ and $100 \%$ of RCA replacement appeared to be more complicated, as shown in Fig. 6(d). The surface was porous and covered with loose crumbly particles, either dust or sources from adhered mortar attached to the coarse RCA after crushing (Fig. 6(b), (c)). The porosity and amount of loose particles covered on the surface depend on the RCA content used in the original concrete.

The ITZ thickness of RCA may range from $10 \mu \mathrm{m}$ to $50 \mu \mathrm{m}$ around the aggregate; this thickness is linked with the degree of hydration and content of adhered mortar (Figs. 6 (c), (d)). The ITZ consists of less unhydrated particle and greater concentration of $\mathrm{Ca}(\mathrm{OH})_{2}$ and ettringite. The microstructure of the specimen also shows that the ITZ of RCA was granular in nature and composed of small pores, micro cracks and fissures, which significantly lowered the bond strength in the area surrounding the mortar matrix

\section{Conclusions}

The engineering and durability performance of RCA was predominantly inferior to conventional concrete, but RCA has been recently gaining wide attention as a construction material. In this study, an experimental program was conducted to investigate the use of RCA as partial replacement of coarse aggregate in concrete production. The replacement level of natural coarse aggregate with RCA reduced the compressive strength of the concrete. However, RCA replacement up to $50 \%$ still achieved medium-high target strength. The water absorption value was directly proportional to the level of RCA replacement. The depth of carbonation increased with increasing percentage of RCA in concrete, which shows that the resistance to carbonation is reduced. Given that carbonation depth is closely related to concrete porosity, incorporating increasingly porous RCA evidently created significantly higher permeable concrete and hence greater carbonation depths. The SEM microstructure of RCA concrete was porous, and its surface was coated with loose particles. Moreover, the ITZ of RCA was composed of numerous tiny pores, micro cracks, and fissures, which relatively weakened the surrounding hardened mortar matrix. Enhancing the quality of concrete by modifying the weak ITZ of RCA and the bulk concrete matrix with mineral admixture, such as fly ash, nano-silica, and meta kaolin, is necessary for future RCA applications. These mineral admixtures behave as micro filler, filling the ITZ between the aggregate surface and the matrix. Additionally, a lack of reliable information on corrosion behavior on RCA has to be established

\section{References}

1. N. Jain, M. Garg, A. K. Minocha, J. Waste Manage, 281048, (2015).

2. W. H. Kwan, M. Ramli, K. J. Kam, M. Z. Sulieman, Construc. Build. Mater. 26 (1), 565-573 (2012).

3. S. Ismail, M. Ramli, Construc. Build. Mater. 44, 464-476 (2013).

4. J. Zhang, C. Shi, Y. Li, X. Pan, C. Poon, Z. Xie, J. Mater. Civ. Eng. 27(11), 04015029 (2015).

5. M. Behera, S.K. Bhattacharyya, A.K. Minocha, R. Deoliya, S. Maiti, Construc. Build. Mater. 68, 501516 (2014).

6. BS 882, British Standards Institution, London, (1992).

7. L. Evangelista, J. de. Brito, Cement Concrete Comp. 29, 397-401 (2007).

8. BS 1881-125, British Standards Institution, London, (1986). 
9. BS EN 12390-3, British Standards Institution, London, (2009).

10. BS 1881-122, British Standards Institution, London, (2011).

11. BS EN 14630, British Standards Institution, London, (2006).

12. W. Li, J. Xiao, C. Shi, C. P. Poon, Adv. Struct. Eng. 18(6), 919-938, (2015).

13. Lotfy, M. Al-Fayez, Cement Concrete Comp. 61, 36-43, (2015).

14. M. Etxeberria, E. Vázquez, A. Marí, M. Barra, Cem. Concr. Res. 35(5), 735-742, (2007).

15. C. Shi, Y. Li, J. Zhang, W. Li, L. Chong, Z. Xie, J. Clean. Prod. 112(1), 1-7, (2015).

16. S. P. Arredondo-Rea, R. Corral-Higuera, J. M. Gomez-Soberon, J. H. Castorena-Gonzalez, V. Orozco-Carmona, J. L. Almaral-Sanchez, Int. J. Electrochem. Sc. 7(2), 1602-1610, (2012).

17. R. V. Silva, J. de Brito, R. K. Dhir, Cement Concrete Comp. 62, 201-217, (2015). 